\title{
The mathematics of spatial transparency and mystery: using syntactical data to visualise and analyse the properties of the Yuyuan Garden
}

\author{
Rongrong $\mathrm{Yu}^{1 *}$, Ning $\mathrm{Gu}^{2}$ and Michael Ostwald ${ }^{2}$
}

\begin{abstract}
Background: One school of thought argues that the Traditional Chinese Private Garden's (TCPG) primary spatial property is that it features both freestanding buildings in space, and spaces freely positions in the landscape, creating a great sense of phenomenal transparency. In contrast, a more traditional interpretation of the TCPG stresses that its primary purpose is to evoke mystery, or provide places of isolation or for retreat.
\end{abstract}

Method: This paper examines these two complex, multi-variable propositions using Space Syntax theory, a method which enables a mathematical analysis of the structural and visual configuration of the spaces in a plan to be undertaken. The methods used for the analysis are variations of the convex space and isovist mapping techniques, and the data is analysed mathematically and then visually using heat-maps. The sixteenth century Yuyuan Garden, one of the most famous TCPGs, is used as a test case for the research.

Results: The results of this study suggest that those features of the TCPG which are associated with mystery are more correlated with its trafficable structure than its visual accessibility. Conversely, the transparency of the TCPG is potentially more related to the directional features of the space than to transparency in isolation.

Conclusion: This paper presents a computational visualisation and analysis method based on the use of Space Syntax techniques for examining vision and movement potential in a complex garden environment. As demonstrated in the example of the Yuyuan garden, the method is effective for analysing and visualising spatial properties beyond the surface level of forms and shapes. Through the application of this method we have been able to provide a new insight into two of the most famous, but poorly understood perceptual properties of TCPGs: transparency and mystery.

\section{Background}

Developed primarily in the sixteenth and seventeenth centuries, traditional Chinese private gardens (TCPG) are today recognised as a special type of landscaped space with a particular set of characteristic properties. In particular, a TCPG is distinguished by its rich spatial arrangement and its associated aesthetic properties, leading to being regarded as having unique experiential qualities (Peng 1986; Tong 1997). But what exactly these characteristics of the TCPG are, has been a subject of on-going debate. For example, some researchers argue that the primary feature of a TCPG is that it creates a space of mystery, evoking a sense of longing and providing opportunities for isolation or retreat (Zou 2013; Conan 1999; Han 2012). In contrast, other scholars have noted that the TCPG often possesses

\footnotetext{
* Correspondence: rongrong.yu@uon.edu.au

${ }^{1}$ Northeast Forestry University, Harbin, China

Full list of author information is available at the end of the article
}

distinctly modern features, including open courtyard gardens and freestanding buildings, which emphasise the phenomenal transparency of its planning (Li 2011). But which of these interpretations are reasonable or appropriate?

In this paper, we use computational and mathematical methods to examine the visual and permeable properties of one of the most famous TCPGs, the sixteenth century Yuyuan Garden (also known as the 'Yu Garden') in Shanghai. The purpose of this research is to compare the relative role played in its planning by spatial properties associated with transparency and mystery. The primary methods used for this analysis are two Space Syntax techniques which, like all such syntactical methods in architectural research, are supported by graph mathematics (Hillier and Hanson 1984; Hillier and Kali 2006; Ostwald 2011; Bafna 2003). However, such is the complexity of the data produced through this comparative analysis that data visualisation plays an important 
role in interpreting the results, and then understanding them in the context of the Yuyuan Garden.

This paper commences with a brief overview to introducing the method that is used for the analysis of transparency and mystery in the Yuyuan Garden. At the conclusion to this section a series of hypotheses are framed about the planar spatial properties that are expected to be found in the Yuyuan Garden, how these will be tested and what mathematical indicators will be used to determine the relative validity of each hypothesis. Thereafter, the holistic results for the Yuyuan Garden, are presented and discussed, using a series of different data visualisation heat-maps, to support their interpretation. Finally, the mathematical properties of several distinct locations in the garden are compared to assess the three hypotheses.

\section{Measuring mystery and transparency}

Two of the more intangible properties of architectural space and form are transparency and mystery. Since the 1980s, a large body of research has been produced which has demonstrated how measures can be derived from an architectural plan to determine levels of efficient movement or surveillance, social control or integration, or predict pedestrian movement patterns, or even relative crime rates and property values (Bhatia et al. 2013; Hillier 1995; Lynch 1960; Ellard 2009). For all of these spatial correlates, robust measures, algorithmic models and visualisation techniques are available. However, for understanding spatial transparency and mystery, the available models mostly provide only indirect indicators of the essence of these properties. For example, it is argued that perceptions of transparency are associated with both visual and permeable properties, respectively, the ability to see through and to move through space (Rowe and Slutzky 1963; Vaughan and Ostwald 2014). Perceptions of mystery can be associated with both the lack of information available about a place (that is, a limited capacity to see or move) and the lack of intelligibility of this information (its complexity due to lack of order) (Hillier 1996; Ostwald and Dawes 2013). Thus, mathematical and computational models of these two spatial properties do exist in syntactical research, but they have not been subjected to the same level of optimisation as several other characteristics of architectural space and form, and they have rarely been used to analyse spaces that are renowned for possessing these properties. One of the reasons for this is that transparency and mystery are, almost by their very nature, more diffuse properties. Yet, they are regularly identified by designers and scholars alike when they describe the most important characteristics of environments (Appleton 1975; Hildebrand 1999). A case in point, in this context, is the TCPG which is celebrated for these properties. The following section describes the Space Syntax method, which is responsible for developing some of the few mathematical tools that are currently available for examining spatial properties associated with mystery and transparency.

\section{Methods \\ Research method - space syntax}

Space Syntax is a collective name for a number of techniques used for analysing the topological properties of space as abstracted into a graph and mathematically analysed (Hillier and Hanson 1984). A graph is a diagrammatic system of representation of sets of nodes and the connections between them (Bondy and Murty 1982). Graph theory is a domain of mathematics which is concerned with the definition, measurement and analysis of graphs. Extensively developed over the last few decades, Space Syntax methods have been widely applied in research in urban planning, architectural design and landscape design, amongst other areas. In particular TCPGs have also been examined using Space Syntax techniques in the past (Yu et al. 2015; Li 2011; Sun 2012).

One of the strengths of the Space Syntax approach is that it provides a way of understanding architectural and urban spatial configurations by translating or abstracting their properties into topological graphs which can then be mathematically analysed (Ostwald 2011; Ostwald and Dawes 2013). The three most widely accepted approaches to abstracting space into a graph for mathematical and visual analysis are convex mapping, axial mapping and isovist mapping (Klarqvist 1992). Variations of these three are used for the present research.

\section{Convex map analysis based on pedestrian accessibility}

To explore the physical accessibility of space - that is, its capacity to be passed over or through - analysis is undertaken of the connectivity relations that exist between spaces. In Space Syntax theory, a visually enclosed or constrained portion of space is called a convex space, because it is in the shape of a convex polygon in which all points are mutually visible to each other (Hillier and Hanson 1984). In the convex space method, an architectural or landscape plan is divided into a set of convex spaces, which are the largest in size and fewest in number (that is, resulting in a highly efficient spatial set). The convex spaces in the map are then abstracted or translated into the nodes of a graph while their connections are converted into the edges of the graph. The connections are usually defined as a property of both adjacency and permeability, that is, the availability of direct access (by way of a door or opening) between two spaces (Peponis and Wineman 2002). These spaces (nodes) and their connections (edges) are collectively termed a convex map or graph (Klarqvist 1992).

The nodes of a convex map possess only basic topological properties, they do not have any extra properties (like area, weight or coordinates) although it is possible to assign labels to them for additional usages (Eloy 2012). The 
connection between the nodes of the graph usually represents the binary property of access or permeability between two nodes (spaces). Accordingly, the convex map graphs are also termed 'adjacency graphs'. Adjacency graphs are commonly illustrated using a carrier node, representing a selected base space (often the major entry to the building or garden). The graph is then drawn like a tree with the carrier as its root. Other nodes are placed (or justified) in ordered levels according to their respective syntactic distance from the carrier. Once graphed in this way, the measures of mean depth $(M D)$, total depth $(T D)$ control value $(C V)$, integration $(i)$ and intelligibility $(I)$ can be derived and then used to analyse the accessibility properties of the plan. These measures are used to identify the least and most integrated spaces (or the order of spaces in terms of integration) in a building. The results (whether expressed as an ordinal rank of spaces or using $i$ values) may be compared to other buildings or different spaces in the same building. Given that spaces usually correspond with the conventional concept 'room' and that the rooms are associated with a number of social functions, these measures are useful for analysing the functional and social configuration of the buildings, urban spaces or large gardens.

The intelligibility value suggests the overall cognitive clarity of the system (the garden in this case) as perceived by a user inside it (Klarqvist 1992; Peponis and Wineman 2002). Intelligibility is defined as the Pearson correlation between integration and the connectivity values of all vertices (the connectivity value of a vertex is the number of directly connected or adjacent vertices to that vertex) (Hillier et al. 1987). Intelligibility is an indication of how easy it is to navigate an environment. A mysterious place will often have a low intelligibility result. The control value is also used to identify spaces in a plan that have a greater role in shaping access to new destinations when a person is navigating through it. Thus a low control value might also suggest a place with heightened mystery.

\section{Isovist analysis based on visual accessibility}

Another way of analysing and visualising the geometric properties of a plan is to create a visual accessibility map, which takes into account the volume of space that can be seen from individual locations in the plan. In Space Syntax theory, a Visual Graph Analysis (VGA) map is used to analyse the spatial properties of a garden (in the present context) in terms of visual accessibility. This style of analysis can be used to explore the spatiovisual properties of a plan, as each point in space has a unique geometrical relationship with its surroundings that defines its singular visual properties. In a floor plan, a unique property of each point is the area visible and accessible from that point, in the shape of a polygon; this is called an isovist (Benedikt 1979). Due to the impracticality of considering all points, the space is typically articulated into a fine grid (ideally corresponding to the size of a human) and isovists of each cell on the grid are then drawn or computed. A graph (called a visibility graph) is then developed with the cells as its nodes and the existence of visibility between cells as its edges (Turner 2001). This visibility graph has a low level of abstraction because every node represents an actual point in space. Therefore, the nodes can possess detailed geometrical properties such as location and isovist characteristics.

Isovist graphs could be regarded as the least abstract of Space Syntax techniques, because the nodes retain a range of geographic properties such as precise size and location. Apart from the graph-based measures, isovist maps also offer a number of pure geometrical measures. These include basic properties like isovist perimeter and area. The latter can also be used to consider concavity but in a different measurement unit. Isovist concavity is calculated by dividing the square of isovist perimeter by the roundness of its area (Franz and Wiener 2008).

Isovists have been used to differentiate visible or hidden locations in a plan (Conroy-Dalton and Bafna 2003). Important isovist measurements for this purpose include drift direction, drift magnitude, occlusivity and jaggedness. Occlusivity is the total length of all edges that are not defined by building surfaces - thus they are the unknown or ill-defined part of the visual experience of a space. Jaggedness $(J)$ is the ratio of Perimeter ${ }^{2}$ to Area. A high $J$ value indicates a more visually complex isovist. High isovist occlusivity indicates a high degree of mystery, in a navigation map this means it may be more difficult to find the way, and in a visual map it means that people may get more visually confused. A high jaggedness indicates a more complex space, both in terms of pedestrian accessibility and visual accessibility.

Drift is the difference between the centre of gravity of an isovist and the location position of the observer. If the difference is very minor, then a space may be quite static. If the difference is more marked, then it might imply a strong sense of directionality, or a strong 'draw', pulling a person through a space. In combination drift magnitude and isovist area can be used to suggest whether a space is phenomenally transparent or literally transparent. While not definitive, a high correlation between isovist drift and area would suggest phenomenal transparency is a major factor in a plan, whereas a low correlation could imply that literal transparency is more important. An inverse correlation might suggest that such a clear pattern does not exist.

\section{Hypothesis framing}

This paper explores the spatial properties of transparency and mystery in the Yuyuan Garden. In order to achieve 
this, various spatial features related to the complexity and mystery related properties of the garden plan, from the point of view of both walking accessibility and visual accessibility, are measured and compared. Complexity refers to the amount of visual information offered by an environment (Scott 1993). Kaplan (1988) states that a high mystery area means that people could learn more if they were to proceed further into the scene. Therefore the concept of complexity and mystery are often linked in the spatial research because they both indicate an intuitive understanding as well as spatial potential. Following the rationale presented in this and the previous sections, three hypotheses, each with associated mathematical indicators, have been framed for testing (Table 1).

\section{Results}

\section{Case study - Yuyuan Garden}

The design principles for TCPGs originated from traditional Chinese culture and literature of Dao (Han 2012; Zou 2013), which applauds a systematic view that nature is a whole cosmos and that everything is a part of it. Zhuangzi, who is the key figure in Daoism, suggested they evoked a transcendental spiritual freedom after achieving the state of forgetting both self and one's surroundings (Zhuangzi $365286 \mathrm{Bc}$ ). Designers of these gardens were mostly scholars and artists, who were willing to create a small peaceful space in an urban plan where the experience of nature was celebrated (Wu 1963). For this reason, the actual area of the TCPG was usually relative small, but by creating wandering pathway, visitors were led to feel that they were in a large scale space, and at one with nature. From the perspective of Feng Shui, this meandering remoteness, formed by the natural topography as well as the cosmological spatial-numerical diagram, emphases a zigzagging movement. These places simulate remote environments where people could easily get lost, but gain happiness and virtue from their experience and process of discovery. In these gardens pathways often led to remote destinations, suggesting the passage of a lifelong journey (Zou 2013).

The Yuyuan Garden is located in the city centre of Shanghai, in southern China. It was built in the sixteenth century and has an area of around 20,000 $\mathrm{m}^{2}$. Parts of the garden were destroyed during the Second World War, although most sections have since been repaired or rebuilt. The Yuyuan garden is well known for its delicate and subtle planning and for its artificial landscape, with water in its centre. It was selected for the present research for three reasons. First, it is one of the most famous TCPGs and it is widely regarded as representing or encapsulating their general characteristics. Second, it has been previously linked to arguments about the importance of transparency and mystery. For instance, $\mathrm{Gu}$ (2013) suggests that the structure of the Yuyuan Garden works to increase the rich experience of the space by extending the walking path, and blocking views of the space using walls and plants, to create a distinct sense of mystery. The third reason is that the Yuyuan garden combines both a modest scale and a level of complex spatial configuration which is appropriate for testing the properties of transparency and mystery.

Testing Hypothesis 1: The sense of mystery in the TCPG is a by-product of structural permeability rather than visual accessibility.

There are two ways of understanding the planning of a TCPG: one is through the pedestrian accessible parts of the plan and the other is by way of its visually accessible parts. To explore the mystery of TCPGs, we commenced with an integration map of both properties of the plan. In this study, two types of data maps are used as input data for the software used for the calculations - UCL Depthmap - the convex map and VGA map. The convex map is based on the connectivity of each space

Table 1 Hypothesis and space measurement

\begin{tabular}{|c|c|c|c|}
\hline Hypothesis & Interpretation & Mathematical properties & Result \\
\hline $\begin{array}{l}\text { The sense of mystery in the TCPG } \\
\text { is a by-product of structural } \\
\text { permeability rather than visual } \\
\text { accessibility. }\end{array}$ & $\begin{array}{l}\text { The network of paths in the TCPG is } \\
\text { very difficult to navigate and } \\
\text { understand, but the spaces that can } \\
\text { be seen from the paths are more } \\
\text { intelligible. }\end{array}$ & $\begin{array}{l}\text { 1) Intelligibility: being the Pearson } \\
\text { correlation between integration and } \\
\text { the connectivity values of all vertices } \\
\text { in the plan graph.2) Control: the } \\
\text { degree by which a space controls } \\
\text { access to its neighbours. }\end{array}$ & $\begin{array}{l}\text { If the hypothesis is true, then: } \\
\text { 1) path-related intelligibility measures } \\
\text { will be lower than vision related } \\
\text { intelligibility.2) path-related control } \\
\text { measures will be lower than vision- } \\
\text { related control. }\end{array}$ \\
\hline $\begin{array}{l}\text { The visual sense of mystery in the } \\
\text { TCPG is heightened by the } \\
\text { complexity of its spaces. }\end{array}$ & $\begin{array}{l}\text { Spaces in the TCPG have both high } \\
\text { levels of visual mystery and of visual } \\
\text { complexity. }\end{array}$ & $\begin{array}{l}\text { 1) Isovist occlusivity: the total length } \\
\text { of all occluded edges in a view- } \\
\text { shed.2) Isovist jaggedness: the ratio of } \\
\text { Perimeter }{ }^{2} \text { to Area in a view-shed. }\end{array}$ & $\begin{array}{l}\text { If the hypothesis is true, then: } \\
\text { There will be a positive correlation } \\
\text { between spatial integration (i) and both } \\
\text { occlusivity }(O) \text { and jaggedness }(J) \\
\text { results. }\end{array}$ \\
\hline $\begin{array}{l}\text { The sense of phenomenal } \\
\text { transparency in the TCPG is a by- } \\
\text { product of the visual pull and } \\
\text { directionality experienced in } \\
\text { major spaces. }\end{array}$ & $\begin{array}{l}\text { The sense of transparency } \\
\text { experienced in large spaces is caused } \\
\text { by spatial irregularity, meaning that } \\
\text { the eye is drawn to look deeper into } \\
\text { the environment. }\end{array}$ & $\begin{array}{l}\text { 1) Isovist area: the area of isovist } \\
\text { polygon.2) Drift magnitude: the } \\
\text { distance from observation point to } \\
\text { centre of mass of isovist polygon. }\end{array}$ & $\begin{array}{l}\text { If the hypothesis is true then: } \\
\text { Drift magnitude will increase in line } \\
\text { with isovist area. }\end{array}$ \\
\hline
\end{tabular}


which is available for accessing; the spaces and linkages between them are input as initial data. While the VGA map is based on the visible boundaries of the space which provides the input data. Figure 1a shows the integration heat-map of the pedestrian component of the garden, and Fig. 1b, the equivalent map of its visual accessibility. The heat-maps in this paper are all produced using UCL Depthmap software. The colour ranges from red to blue, where the red colour represents a high value and the dark blue represents a low value. For instance, in Fig. 1a, the red colour identifies the pathways with the highest integration value, which are the most accessible or likely to be used by people. The dark blue is the least accessible, or statistically least likely to be used. From that figure, we can see that in terms of pedestrian accessibility, the most integrated part is the centre area around the water, and the least integrated parts are to the southern and northeastern corners. For the visual accessibility (Fig. 1b), the most transparent parts of the plan are in the middle area as well, and least are the northwest part behind the artificial hill and also the south corner which is blocked by solid walls.

To further understand the mystery and openness of the TCPG plan, two variables are measured: intelligibility and control. These two variables were selected because past research (see the previous section) has identified their significance in terms of the navigational properties of a space, which in turn reflect the sense of either clarity or mystery or openness or enclosure. Intelligibility is the Pearson correlation between integration and the connectivity values of all vertices in the plan graph. It provides a measure of how difficult it will be for people to navigate through a space. While control is the degree to which a space limits access to its neighbours, which suggests a greater impact for a space in shaping new destinations when a person is navigating through it.

Table 2 shows the holistic intelligibility and control values of both pedestrian accessibility and visual accessibility maps for the entire garden. From the results, intelligibility is higher in the visual map than in the walking map $(0.763>0.570)$, which means that it is easier to understand the space visually, suggesting a level of higher mystery in terms of pedestrian accessibility. Because in this application intelligibility is effectively a normalised value (in the range between 0 and 1), a simple reading of the difference is that it is in the order of around $16 \%$ lower for pedestrian accessibility that visual accessibility. This can be explained as the fact that a person may get lost or experience difficulty accessing different places in the garden that can be more readily seen. The visual accessibility map also has a higher control value than the pedestrian accessibility map (1.000 $>0.929$ ). While this difference is marginal, the overall control value is stronger visually, which implies again

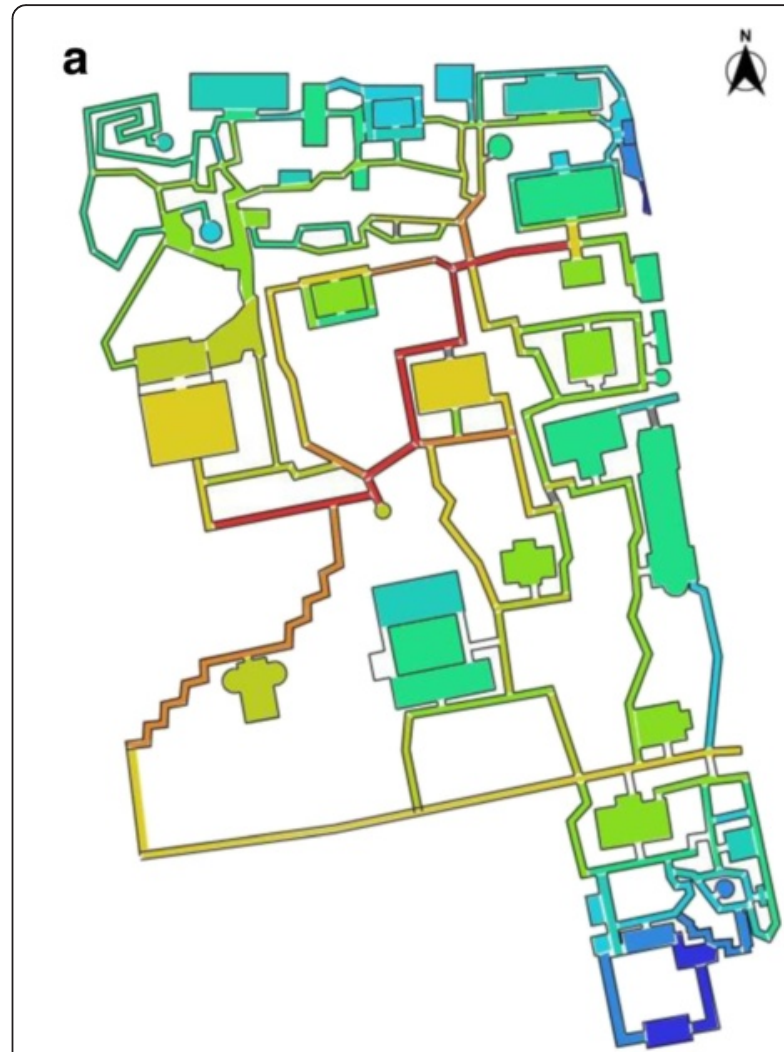

b

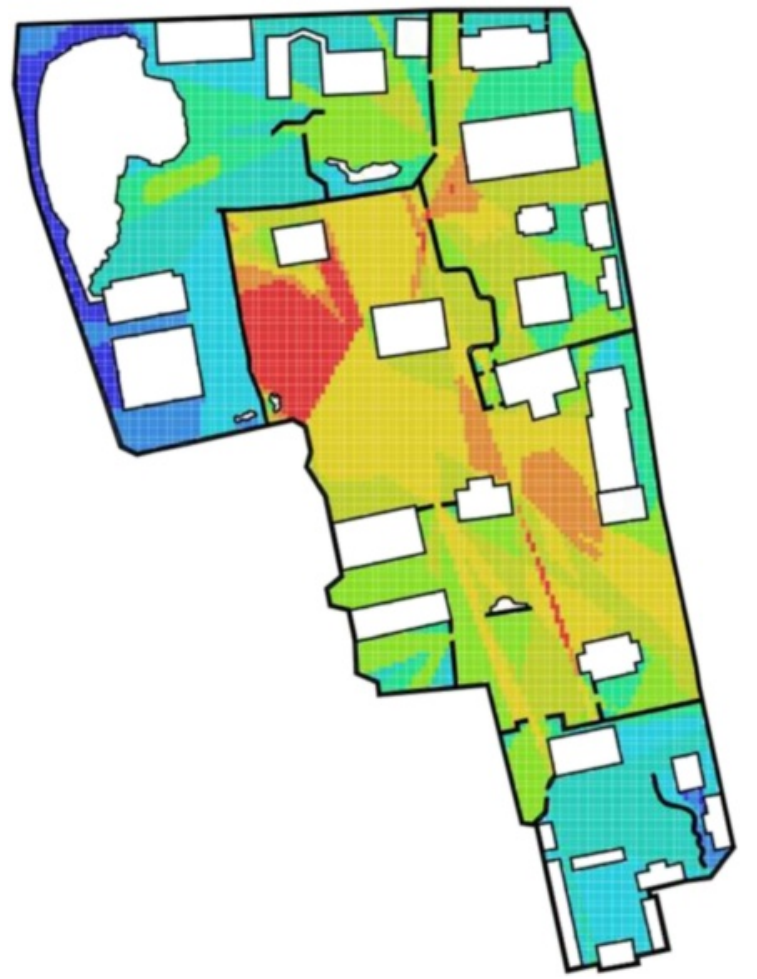

Fig. 1 Yuyuan Garden, (a) integration heat-maps of pedestrian accessibility, (b) visual accessibility 
Table 2 Intelligibility and control of convex map and VGA map, for the Yuyuan Garden

\begin{tabular}{lcc}
\hline & Intelligibility & Control \\
\hline Pedestrian accessibility map & 0.570 & 0.929 \\
Visual accessibility map & 0.763 & 1.000 \\
\hline
\end{tabular}

that there is slightly more mystery in the walking path. However, the difference is so marginal, that it might support Hypothesis 1, but it must be combined with the results for intelligibility to reach a more emphatic conclusion.

Testing Hypothesis 2: The visual sense of mystery in the TCPG is heightened by the complexity of its spaces.

To examine the second hypothesis the relationship between three measures - integration, isovist occlusivity and jaggedness - was examined. Figure 2 shows three locations in the garden which were selected for detailed analysis on the basis of the total set of integration results in the visual integration map. Because an infinite number of possible comparison points can be determined and generated, locations were chosen to represent and compare the two extremes in the data. However, a visual assessment of the heat-map was undertaken which revealed that the highest integration area (red area) is a single, relatively clear location in the centre, but there are two low integration areas in different parts of the

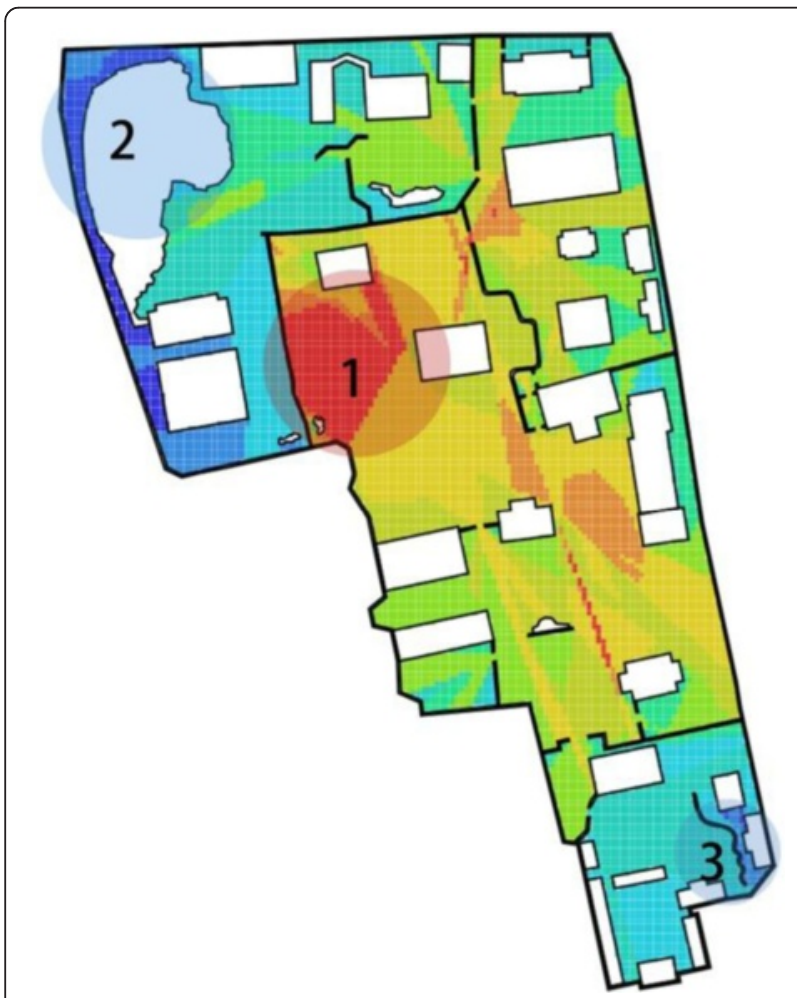

Fig. 2 Yuyuan Garden, VGA map showing zones 1-3 garden but with similar values. These two low integration zones, 2 and 3, were therefore both included and are represented in blue circles in the figure.

Figure 3 shows the isovist polygon area found at each of these three locations. As we can see in the figure, the high integration area has a larger isovist area than the two low integration areas. This indicates that the high integration area has an open visual property that could be visually permeable to a relatively larger area. Conversely, the low integration spaces are more likely to be visually blocked or hidden by walls, hills or buildings.

In the next step, isovist occlusivity, area and perimeter heat-maps were produced for the garden (Fig. 4) and for the three zones, local isovist occlusivity and jaggedness values were measured (Table 3). A high occlusivity value means that there are less defined, and thereby potentially more confusing, properties to a space. Jaggedness represents the degree of complexity of the view from a particular space. The results for these two measures, for local zones, (Table 3) show a correlation between high integration and high occlusivity and jaggedness values. This implies that there is a positive relation between the three, or that significant spaces (high $i$ values) in the Yuyuan Garden are both spatially rich and complex (high $J$ values) and are also high in mystery ( $O$ values). Another way of looking at this result is that the most important spaces in the TCPG, in terms of social copresence and navigation, are also the most complex and mysterious.

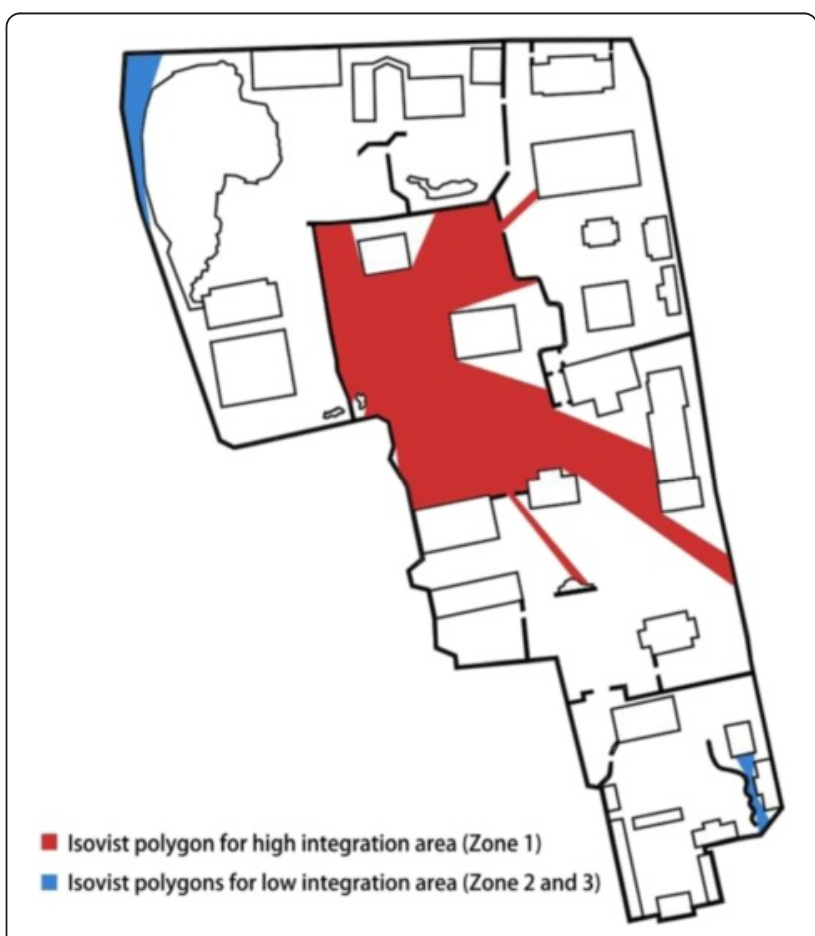

Fig. 3 Yuyuan Garden, isovist polygons for each zone 


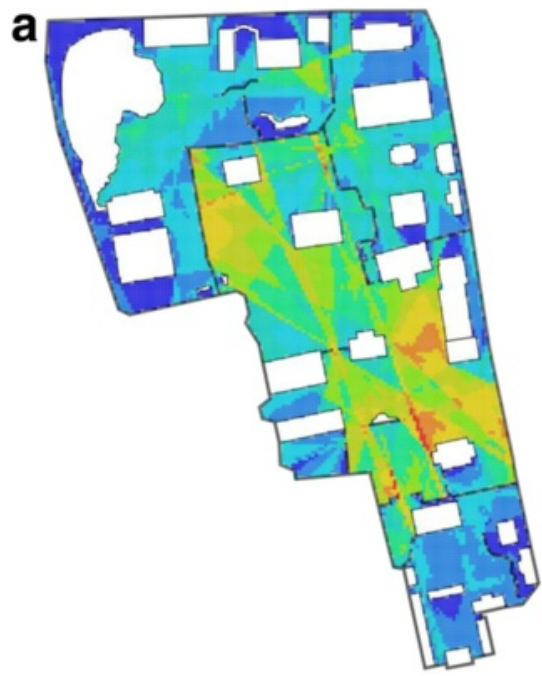

b

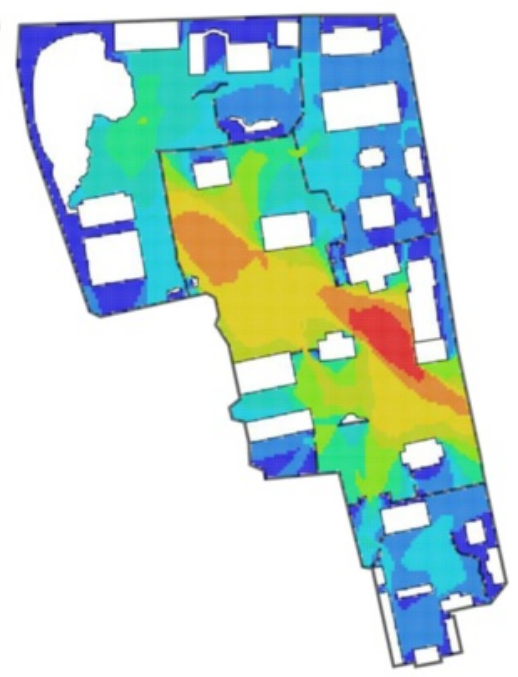

C

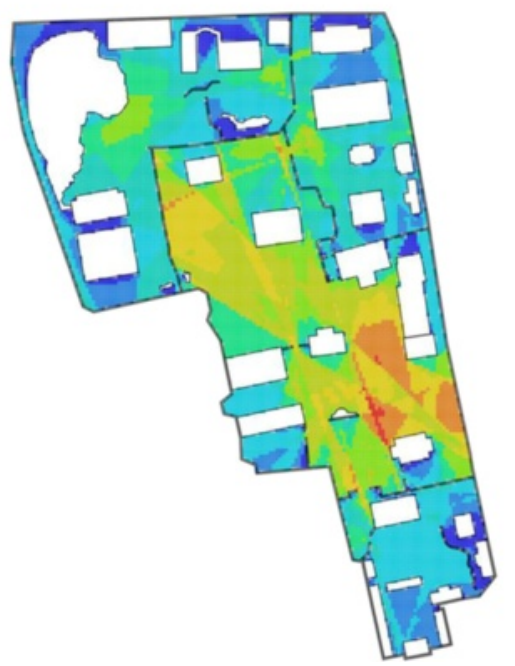

Fig. 4 Yuyuan Garden, heat-map of (a) isovist occlusivity, (b) isovist area and (c) perimeter
Table 3 Yuyuan Garden, VGA zone results of isovist occlusivity and jaggedness

\begin{tabular}{lcc}
\hline Isovist & Occlusivity $(O)$ & Jaggedness $(J)$ \\
\hline Zone 1 & 273.657 & 84.726 \\
Zone 2 & 23.063 & 61.356 \\
Zone 3 & 24.278 & 40.074 \\
\hline
\end{tabular}

Testing of Hypothesis 3: The sense of transparency in the TCPG is a by-product of the visual pull and directionality experienced in major spaces.

For the present paper, the spatial property of transparency is measured using a combination of isovist area and drift magnitude. A larger isovist area means that the visible portion of the space is more extensive, and therefore it potentially offers greater opportunity to see, increasingly the literal sense of transparency. However, transparency is also a factor of the extent to which a person is drawn through space, a concept known as phenomenal transparency. Thus, a very large space might offer an extensive viewshed, but it could be of a static or dull space, whereas a slightly smaller space could draw the viewer's eye to look more deeply into an environment, thereby increasing the sense of transparency (Rowe and Slutzky 1963; Ellard 2009). This latter quality is associated with the isovist property, drift magnitude.

The VGA analysis results for isovist area and drift magnitude show that area is more differentiated across the garden, which may be visualised by examining the red/yellow portions of Fig. 5. In contrast, drift magnitude is less differentiated, implying a more consistent or evenly dispersed sense of phenomenal transparency. When the equivalent results for the three test zones are considered in isolation (Table 4), there is a general trend of increasing integration, with increasing area and increasing drift, but the correlation is not linear. Zone 1 is 17.76 times larger in area than zone 2, which is in turn, 3.59 times larger than zone 3. For drift magnitude the result for zone 1 is 5.01 times larger than zone 2, and zone 2 is in turn, 6.08 times higher than zone 3 . As the overall heat-map visualisation of this data implies, phenomenal transparency and literal transparency both work together to shape the experience of the space, but the former is more diverse than the latter. Another way of stating this is that, the transparent properties of the space of the TCPG are consistently shaped by phenomenal transparency, the knowledge that further passage is possible, but that literal transparency only dominates specific parts of the garden.

\section{Discussion and conclusion}

This paper presents a computational visualisation and analysis method based on the use of Space Syntax techniques for examining vision and movement potential in a complex garden environment. As demonstrated in the 


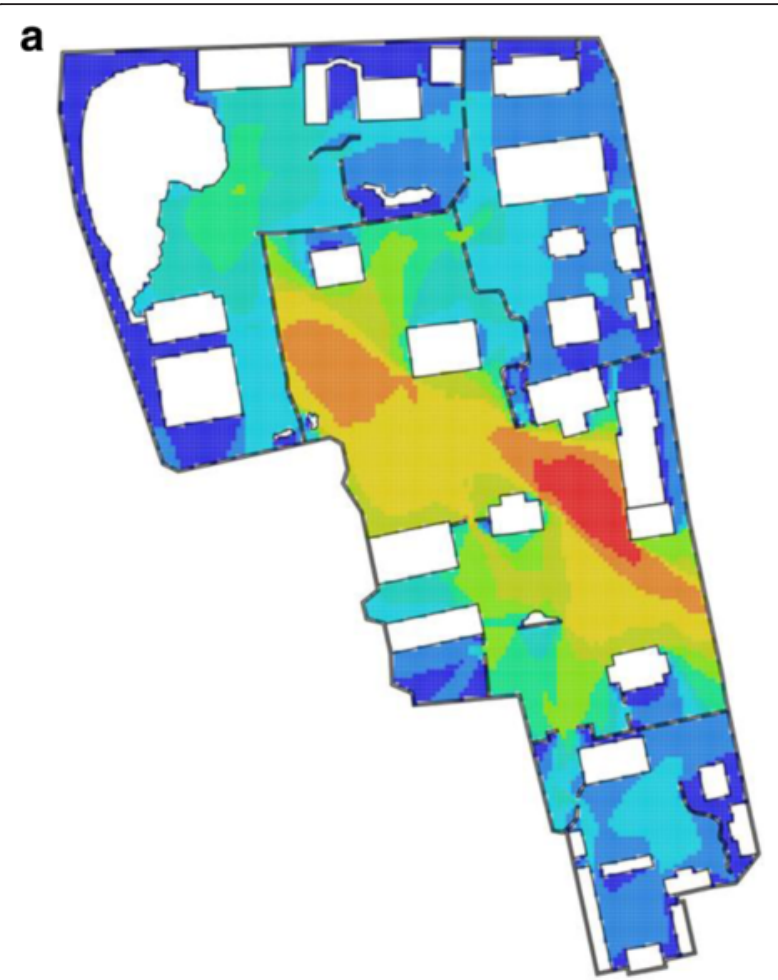

b

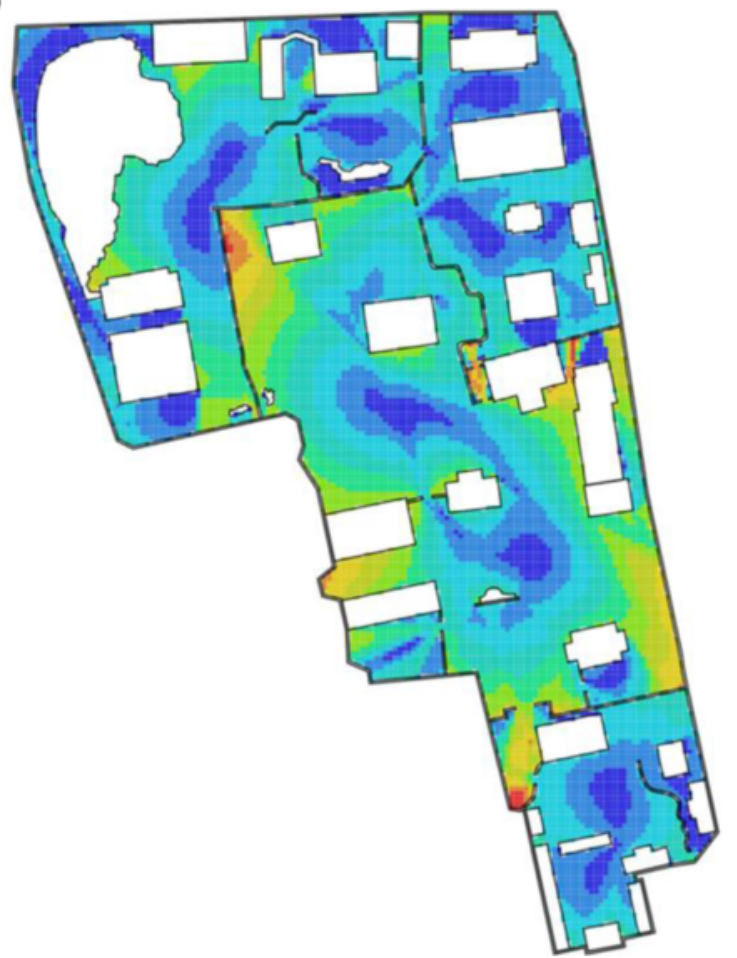

Fig. 5 Yuyuan Garden, heat-map of (a) isovist area and (b) isovist drift magnitude
Table 4 V Yuyuan Garden, VGA zone results of isovist area and drift magnitude

\begin{tabular}{lrc}
\hline Isovist & Area $(A)$ & Drift magnitude $\left(D_{M}\right)$ \\
\hline Zone 1 & 2739.676 & 23.416 \\
Zone 2 & 154.225 & 4.666 \\
Zone 3 & 42.956 & 0.684 \\
\hline
\end{tabular}

example of the Yuyuan garden, the method is effective for analysing and visualising spatial properties beyond the surface level of forms and shapes. Through the application of this method we have been able to provide a new insight into two of the most famous, but poorly understood perceptual properties of TCPGs; transparency and mystery.

This paper has several practical and methodological limitations that are important to take into account. First, the paper is focussed only on two-dimensional representations of space, effectively considering only the plans of the Yuyuan Garden, not its three-dimensional form. However, the analysis does take into account contours when calculating visual accessibility. Thus, any artificial hills in the garden which are over $1.7 \mathrm{~m}$ in height do restrict vision. Second, the analysis doesn't differentiate between some properties of the gardens. For example, low-lying plants and small garden walls are treated in the same way, although in reality they are different things. In practical terms though, both of these features impede movement, but not vision; we can see over them, but moving through them is more difficult. Third, in both a cognitive and a phenomenological sense, the experience of spatial mystery and transparency is far more complex and contingent than the method adopted in this paper might suggest. The techniques used in the present paper provide a statistical and geometrically mapped generalisation of spatial and visual properties but they do not directly take into account individual spatial preferences of behaviours. Nevertheless, research cited in this paper does map these mathematical properties and results directly to human attitudes and activities, so the method adopted for this research is not without appropriate foundations in human perceptual and behaviour studies, although this aspect of the research is not developed in detail in the paper.

The results of this research show that for the Yuyuan Garden, mystery is potentially more a product of structural permeability than visual accessibility. This confirms the first hypothesis. In practical terms the result means that, for example, when navigating in the Yuyuan Garden, people will often find that spaces or objects which they can see, are much more difficult to reach. Furthermore, this quality of spatial mystery is compounded and reinforced by the complexity of the garden space. This confirms the second hypothesis. 
The third hypothesis suggests that the sense of transparency in the Yuyuan Garden is a by-product of the visual 'pull' and 'directionality' experienced in major spaces. This result is also broadly supported by the data, but the relationship between area and transparency requires a more extensive study to produce a definitive outcome. The limited data used here suggests that there is a relatively consistent level of phenomenal transparency, which may be either heightened or undermined, by a less consistent level of literal transparency. Thus, for example, a space may be relatively large, and a person may be able to see a long way through it, but they are not always drawn to move through that space in a consistent, or even practical way.

Ultimately, this paper uses quantitative methods to examine a previously qualitative subject of debate focused on seemingly intangible characteristics. The primary contribution of this research is to enhance our understanding of the spatial features of the TCPG, as represented in the Yuyuan Garden case study. The second contribution is to demonstrate that syntactical analytical methods are appropriate for examining the spatial features in complex landscape designs. The third is to emphasise the usefulness of data visualisation techniques for intuitively interpreting complex results. Future research arising from this paper will enlarge the sample size of the study to increase the generalizability of the results and include other spatial features in the TCPGs into the analysis.

\section{Competing interests}

The authors declare that they have no competing interests.

\section{Authors' contribution}

$\mathrm{RY}, \mathrm{NG}$ and $\mathrm{MO}$ carried out the molecular genetic studies, participated in the sequence alignment and drafted the manuscript. All authors read and approved the final manuscript.

\section{Author details}

${ }^{1}$ Northeast Forestry University, Harbin, China. ${ }^{2}$ The University of Newcastle, Newcastle, Australia.

Received: 29 October 2015 Accepted: 26 January 2016

Published online: 16 February 2016

\section{References}

Appleton, J. (1975). The experience of landscape. London: Wiley.

Bafna, S. (2003). Space syntax: a brief introduction to its logic and analytical techniques. Environment and Behavior, 35, 17-29.

Benedikt, M. L. (1979). To take hold of space: isovists and isovist fields. Environment and Planning B: Planning and Design, 6, 47-65.

Bhatia, S., Chalup, S. K., \& Ostwald, M. J. (2013). Wayfinding: a method for the empirical evaluation of structural saliency using $3 \mathrm{~d}$ isovists. Architectural Science Review, 56, 220-231.

Bondy, J. A., \& Murty, U. S. R. (1982). Graph theory with applications. New York: Elsevier Science Publishing.

Conan, M. (1999). Perspectives on garden histories. Dumbarton Oaks: Research Library and Collection.

Conroy-Dalton, R., Bafna, S. (2003). The syntactical image of the city: a reciprocal definition of spatial elements and spatial syntaxes proceedings of the 4th International Space Syntax Symposium, London.

Ellard, C. (2009). You are here. New York: Random House.
Eloy, S. (2012). A transformation grammar-based methodology for housing rehabilitation: meeting contemporary functional and Ict requirements (Phd Thesis). Lisbon: University Of Technology.

Franz, G. \& Wiener, J. M. (2008). From space syntax to space semantics: a behaviorally and perceptually oriented methodology for the efficient description of the geometry and to-pology of environments. Environment and Planning B: Planning and Design, 35, 574-592.

Gu, K. (2013). The Private Garden Of Jiangnan. Beijing: Thsinghua University Press.

Han, C. (2012). The aesthetics of wandering in the Chinese literati garden. Studies in the History of Gardens \& Designed Landscapes: An International Quarterly, 32, 296-301.

Hildebrand, G. (1999). Origins of architectural pleasure. Oakland: University Of California Press.

Hillier, B. (1995). Space is the machine. Cambridge: Cambridge University Press. Hillier, B. (1996). Space is the machine : a configurational theory of architecture. Cambridge: Cambridge University Press.

Hillier, B., Hanson, J. (1984). The Social Logic Of Space. Cambridge: Cambridge University Press.

Hillier, B., Hanson, J., \& Graham, H. (1987). Ideas are things, an application of space syntax to discovering house genotypes. Environment and Planning $B$ : Planning and Design, 14, 363-385.

Hillier, B., \& Kali, T. (2006). Space syntax: the language of museum space. In M. Sharon (Ed.), A companion to museum studies. London: Black Well.

Kaplan, S. (1988). Perception and landscape: conceptions and misconeptions. In J. L. Nasar (Ed.), Environmental aesthetics. Cambridge: University Of Cambridge.

Klarqvist, B. (1992). A space syntax glossary. Arkitekturforskning.

Li, Z. (2011). Visual Perception Of Traditional Garden Space. In Suzhou, China: A Case Study With Space Syntax Techniques. 2011 19th International Conference On Geoinformatics, 24-26 June 2011 (pp. 1-4).

Lynch, K. (1960). The image of the city. Cambridge: MIT Press.

Ostwald, M. (2011). The mathematics of spatial configuration: revisiting, revising and critiquing justified plan graph theory. Nexus Network Journal, 13, 445-470.

Ostwald, M., \& Dawes, M. (2013). Differentiating between line and point maps using spatial experience: considering Richard Neutra's Lovell House. Nexus Network Journal, 15, 63-81.

Peng, Y. (1986). Analysis of Chinese classical garden. Beijing: China Architecture and Building Press.

Peponis, J., \& Wineman, J. (2002). Spatial structure of environment and behavior. In R. Bechtel \& A. Churchman (Eds.), Handbook of environmental psychology. New York: Wiley.

Rowe, C., Slutzky, R. (1963). Transparency: literal and phenomenal. Cambridge: The Mit Press On Behalf Of Perspecta.

Scott, S. (1993). Complexity and mystery as predictors of interior preferences. Journal of Interior Design, 19, 25-33.

Sun, P. (2012). The contrast interpretation of chinese classical gardens between space syntax theory and traditional theories-the space research of Chengde mountain resort. Doctoral Dissertation. Beijing: Beijing Forestry University.

Tong, J. (1997). Glimpses of gardens in Eastern China. Beijing: Architecture and Building Press.

Turner, A. (2001). Depthmap: a program to perform visibility graph analysis. Atlanta: 3rd International Space Syntax Symposium.

Vaughan, J., \& Ostwald, M. J. (2014). Measuring the significance of façade transparency in Australian regionalist architecture: a computational analysis of 10 designs by Glenn Murcutt. Architectural Science Review, 57, 249-259.

Wu, N. I. (1963). Chinese and Indian Architecture: the City of Man, the Mountain of God, and the Realm of the Immortals. New York: G. Braziller.

Yu, R., Ostwald, M., Gu, N. (2015). Parametrically generating new instances of traditional Chinese private gardens that replicate selected socio-spatial and aesthetic properties. Nexus Network Journal, 1-23. First online: 01 August 2015, doi:10.1007/s00004-015-0263-7.

Zhuangzi 365-286 Bc. Zhuangzi Jin Zhu Jin Shi. Hongkong: Zhonghua Shuju.

Zou, H. (2013). The Idea of Labyrinth (Migong) in Chinese Building Tradition. Journal of Aesthetic Education, 46, 80-95. 